\title{
Downregulation of CCR5 inhibits the proliferation and invasion of cervical cancer cells and is regulated by microRNA-107
}

\author{
LI-FAN CHE, SU-FANG SHAO and LI-XIN WANG
}

Department of Gynecology and Obstetrics, Yi-Du Central Hospital of Weifang, Weifang, Shandong 262500, P.R. China

Received July 24, 2014; Accepted September 15, 2015

DOI: 10.3892/etm.2015.2911

\begin{abstract}
Cervical cancer is among the most prevalent forms of cancer worldwide. C-C chemokine receptor type 5 (CCR5) is hypothesized to be a key functional protein involved in tumorigenesis. However, the role of CCR5 in cervical cancer remains unclear. Reverse transcription-quantitative polymerase chain reaction and western blot analysis were used to evaluate the mRNA and protein expression levels of CCR5 in human cervical carcinoma tissues. Furthermore, a small interfering RNA was employed to knockdown CCR5 in HeLa and C33A cells. MTT, colony formation and Transwell assays were performed to determine the effects of this knockdown on cell viability, proliferation and invasion. In addition, micro RNA (miR)-107 was identified as a potential candidate regulator of CCR5 using miR prediction algorithms, and the effects of miR-107 and its antisense miR on CCR5 mRNA expression were determined. The results of the present study indicated that CCR5 is overexpressed in human cervical cancer tissues compared with adjacent normal tissues, and its downregulation inhibits cervical cancer cell growth and proliferation. Furthermore, the downregulation of CCR5 appears to suppress cervical cancer cell invasion. Finally, the tumor suppressor miR-107 was able to directly target CCR5 and inhibit its expression. These results suggest that the upregulation of CCR5, which is inhibited by miR-107, may play a carcinogenic role in cervical cancer and could provide a novel therapeutic target in the future.
\end{abstract}

\section{Introduction}

Cervical cancer is the second most prevalent type of cancer among women worldwide (1), and is a complex disease involving numerous oncogenes or the abnormal expression of tumor suppressors $(2,3)$. Currently, the phosphoinositide

Correspondence to: Dr Li-Fan Che, Department of Gynecology and Obstetrics, Yi-Du Central Hospital of Weifang, 4138 Linglong Road, Weifang, Shandong 262500, P.R. China

E-mail: chelifan23000@163.com

Key words: C-C chemokine receptor type 5, human cervical cancer, invasion, microRNA-107
3-kinase(PIK3)/protein kinase B signaling pathway is considered crucial to the pathogenesis of cervical cancer. The PIK3 catalytic subunit $\alpha$ gene is upregulated in cervical cancer due to the amplification of the chromosome 3q26.3 locus (4). In addition, tumor suppressor genes, such as phosphatase and tensin homolog, may be downregulated due to genetic mutations or deletions, which contribute to the development of cervical cancer (5). However, the precise molecular mechanisms underlying the pathogenesis of cervical carcinogenesis remain unclear. Therefore, it is crucial to identify specific molecular markers and mechanisms for use in cervical cancer detection.

C-C chemokine receptor type 5 (CCR5) belongs to the chemokines, a family of structurally-related proteins that were initially recognized as mediators of chemotaxis and cellular homing (6). This family is loosely divided into three groups: Homeostatic/constitutive chemokines, inflammatory/inducible chemokines $(7,8)$ and dual function chemokines. To date, CCR5 has been demonstrated to be involved in a variety of biological processes, including tumor development. For example, the expression of chemokine (C-C motif) ligand 5 (CCL5), a ligand that binds with CCR5, correlates with breast cancer stage (9) and is associated with enhanced melanoma formation in nude mice (10). Furthermore, treatment with a CCL5 antagonist was observed to decrease tumor growth in a breast cancer model (11). Ng-Cashin et al demonstrated that CCR5 knockout was able to inhibit local tumor growth and improved responses to cancer vaccines in mice (12). In addition, van Deventer et al showed that the expression of CCR5 in stromal cells promoted pulmonary metastasis (13). However, few studies have investigated the association between CCR5 and cervical cancer development.

The aim of the present study was to investigate the expression of CCR5 in human cervical cancer cells, and to evaluate the effect of CCR5 knockdown on the viability, colony formation and invasiveness of the cells. Furthermore, the potential of micro RNA (miR)-107 as a regulator of CCR5 expression in the cervical cancer cells was evaluated.

\section{Materials and methods}

Human tissue samples. A total of 28 pairs of human cervical cancer and adjacent normal tissues were obtained from the Department of Gynecology, Yi-Du Central Hospital of Weifang, (Weifang, China). Informed consent was obtained 
from all patients. The majority of the cancer was stage IIa or lower according to the International Federation of Gynecology and Obstetrics (FIGO) staging system (14). Histologically, all included biopsies were squamous cell carcinoma. All use of human specimens was approved and supervised by the Ethics Committee of Jinan Maternity and Child Care Hospital. The specimens were frozen in liquid nitrogen and stored at $-80^{\circ} \mathrm{C}$ until required.

$R N A$ isolation and reverse transcription-quantitative polymerase chain reaction ( $R T-q P C R)$. Total RNA was isolated using Invitrogen TRIzol reagent (Thermo Fisher Scientific, Inc., Waltham, MA, USA) according to the manufacturer's protocol. Oligo (dT) primers and M-MLV reverse transcriptase (Promega Corporation, Madison, WI, USA) were applied to reverse transcribe the $1 \mu \mathrm{g}$ total RNA into cDNA. qPCR was performed to detect the CCR5 mRNA expression level using a SYBR Premix Ex Taq ${ }^{\mathrm{TM}}$ kit (Takara Bio, Dalian, China) according to the manufacturer's protocol. $\beta$-actin was used as the reference gene. qPCR cycling was performed using the iQ5 real-time PCR detection system (Bio-Rad Laboratories, Inc., Hercules, CA, USA), under the following conditions: Denaturing at $94^{\circ} \mathrm{C}$ for $4 \mathrm{~min}$, followed by 40 cycles of amplification including $94^{\circ} \mathrm{C}$ for $60 \mathrm{sec}, 58^{\circ} \mathrm{C}$ for $60 \mathrm{sec}$, and $72^{\circ} \mathrm{C}$ for $60 \mathrm{sec}$. Primers used for the qPCR were as follows: CCR5 forward, 5'-GAGACTCTTGGGATGACGC-3' and reverse, 5'-GTTTGGCAATGTGCTTTTG-3'; and $\beta$-actin forward, 5'-TGCGTGACATTAAGGAGAAGC-3' and reverse, 5'-TCC ATGCCCAGGAAGGAA-3' (Genewiz, Inc., Beijing, China). All qPCR experiments were conducted in triplicate.

Western blot analysis. Cells were homogenized in radioimmunoprecipitation assay (RIPA) lysis buffer (BioVision, Inc., Milpitas, CA, USA) in the presence of $1 \%$ (v/w) protease inhibitor cocktail (Pierce Biotechnology, Inc., Rockford, IL, USA). Total protein was isolated and, following the measurement of its concentration using a Bicinchoninic acid assay (Qcbio Science $\&$ Technologies, Co., Ltd., Shanghai, China), $20 \mu$ g protein was separated by $10 \%$ sodium dodecyl sulfate-polyacrylamide gel electrophoresis (ZhiYou Biotechnology Co., Ltd., Guangzhou, China) and blotted onto nitrocellulose membranes (EMD Millipore, Billerica, MA, USA). The membranes were subsequently incubated with polyclonal rabbit anti-human CCR5 antibody (1:1,000; ab65850; Abcam, Cambridge, MA, USA) and monoclonal rabbit anti-human $\beta$-actin antibody $(1: 1,000$; ab181602; Abcam) in blocking solution overnight at $4^{\circ} \mathrm{C}$. After washing five times with phosphate-buffered saline solution, the membranes were probed with a secondary horseradish peroxidase-conjugated goat anti-rabbit IgG antibody (1:1,000; ab150077; Abcam) for $2 \mathrm{~h}$ at room temperature. The relative amount of protein was normalized to $\beta$-actin and analyzed with a Gel-Pro Analyzer, version 4.0 (Media Cybernetics, Inc., Rockville, MD, USA).

Small interfering RNA (siRNA) for CCR5 knockdown. siRNA used for the knockdown of CCR5 (siRNA-CCR5) was purchased from Chang Jing Bio-Tech, Ltd. (Changsha, China). The CCR5 knockdown siRNA sequence was as follows: siRNA-CCR5 (top), 5'-GATCCGTCCAATCTATGACAT CAATTCAAGAGATTGATGTCATAGATTGGACTTTTT
TGGAAGAATTCA-3' and siRNA-CCR5 (bottom), 5'-AGC TTGAATTCTTCCAAAAAAGTCCAATCTATGACA

TCAATCTCTTGAATTGATGTCATAGATTGGACG-3'. A scrambled siRNA (siRNA-NC) was used as a control.

Cell culture and transfection. Human cervical cancer cell lines HeLa and C33A (ZhiYou Biotechnology) were maintained in RPMI-1640 medium (Thermo Fisher Scientific, Inc.) supplemented with $10 \%(\mathrm{v} / \mathrm{v})$ heat-inactivated fetal bovine serum (FBS), $100 \mathrm{IU} / \mathrm{ml}$ penicillin and $100 \mu \mathrm{g} / \mathrm{ml}$ streptomycin (Sigma-Aldrich, St. Louis, MO, USA) at $37^{\circ} \mathrm{C}$ in a humidified atmosphere with $5 \% \mathrm{CO}_{2}$. Transfection was performed using Lipofectamine 2000 reagent (Thermo Fisher Scientific, Inc.) following the manufacturer's protocol.

MTT and colony formation assays. Transfected cells were plated in 96-well plates at a density of 5,000 cells/well. At $48 \mathrm{~h}$ after transfection, the cells were incubated with MTT for $4 \mathrm{~h}$ at $37^{\circ} \mathrm{C}$. The cells were then agitated with MTT solvent on an orbital shaker at $377 \mathrm{x} \mathrm{g}$ for $10 \mathrm{~min}$ in the dark at room temperature (Thermo Fisher Scientific, Inc.). The absorbance was measured at $570 \mathrm{~nm}$ using a spectrophotometer (M5/M5a; Xinhua, Guangdong, China). In the colony formation assay, cells were seeded into a 12-well plate at a density of 200 cells/well, with a change of medium every 3 days. After $\sim 10$ days, the majority of the cell clones contained $>50$ cells. The clones were washed with 1X phosphate-buffered saline and stained with crystal violet (Yuanye Bio-Technology, Co., Ltd., Shanghai, China) for $\sim 5 \mathrm{~min}$.

Cell invasion assay. Invasion assays were performed in 24-well Transwell chambers (Corning Incorporated, Corning, NY, USA). The upper compartments of the chambers were filled with $100 \mu 1$ pre-chilled serum-free RPMI-1640 mixed with Matrigel (1:7; BD Biosciences, Franklin Lakes, NJ, USA). The Matrigel remained at room temperature for $4 \mathrm{~h}$ for solidification. Subsequently, $5 \times 10^{4} \mathrm{HeLa}$ cells or $8 \times 10^{4} \mathrm{C} 33 \mathrm{~A}$ cells were trypsinized, washed and resuspended in serum-free RPMI-1640, then seeded in the upper chamber. An additional $500 \mu 1$ RPMI-1640 containing 10\% FBS was added to the lower chamber as a chemoattractant. The chambers were incubated at $37^{\circ} \mathrm{C}$ in $5 \% \mathrm{CO}_{2}$ for $24 \mathrm{~h}$ (HeLa cells) or $48 \mathrm{~h}$ (C33A cells), then fixed in $100 \%$ methanol. Fixed cells were stained with crystal violet and the number of invasive cells was counted. Five random fields in each chamber were analyzed. Assays were performed in triplicate.

Identification of a CCR5-targeting miRNA using multiple miRNA target prediction algorithms. In order to identify a sequence potentially capable of inhibiting the expression of CCR5, a number of miRNA target prediction software packages were used, namely Targetscan (http://www.targetscan.org), microRNA.org, DIANA (http://diana.imis.athena-innovation. gr/DianaTools/index.php?r=microT_CDS/index) and miRwalk (www.umm.uni-heidelberg.de/apps/zmf/mirwalk/).

Luciferase assay of the effect of miR107 on CCR5 expression. A pcDNA3/enhanced green fluorescent protein (EGFP)-CCR5-3' untranslated region (UTR) vector and the mutant pcDNA3/EGFP-CCR5-3'UTR, in which a 
A

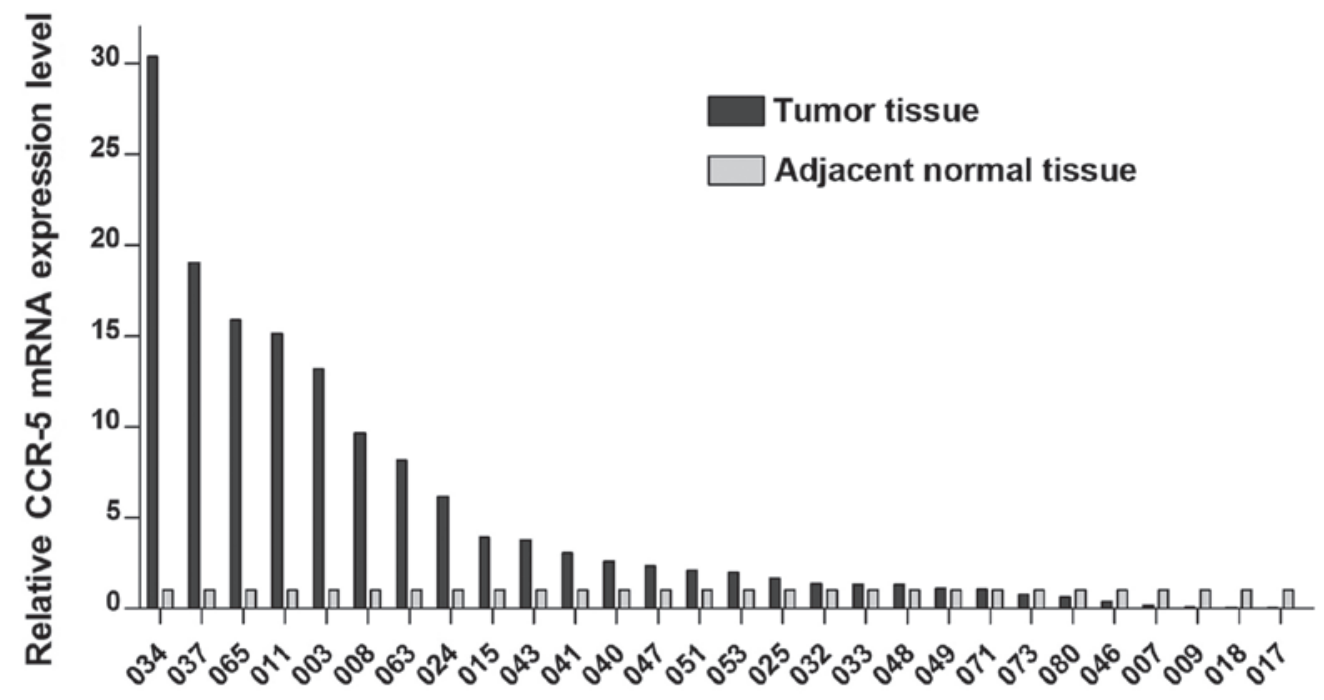

B

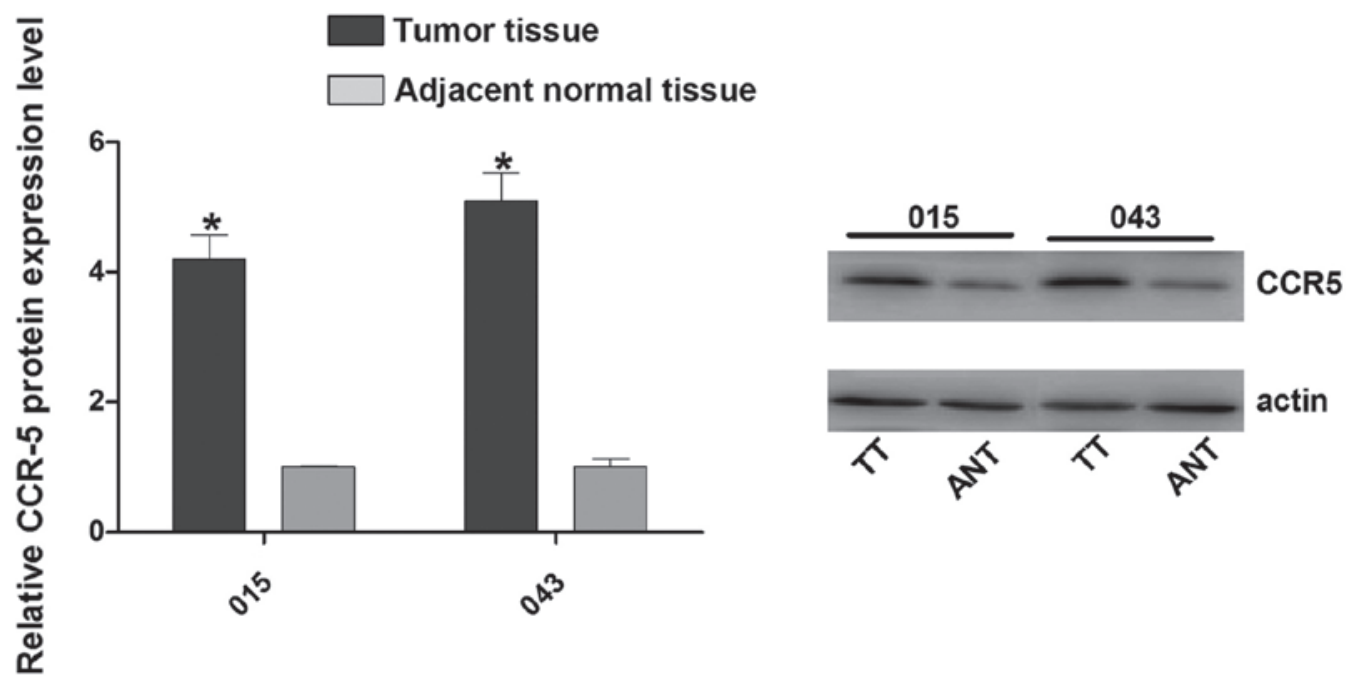

Figure 1. Relative CCR5 mRNA and protein expression levels in cervical carcinoma tissues. (A) CCR5 mRNA expression level in 28 pairs of cervical tumor tissues (TT) and matched adjacent normal tissues (ANT) was determined using reverse transcription-quantitative polymerase chain reaction. GAPDH was used as an endogenous reference gene. (B) Representative protein expression level of CCR5 in cervical tissues is shown in samples 015 and 043 . "P $<0.05$ vs. ANT. CCR5, C-C chemokine receptor type 5.

number of nucleotides within the binding sites were mutated, were purchase from ZhiYou Biotechnology Co., Ltd. For the luciferase reporter assay, the HeLa cells were co-transfected with a miR-107 mimic (Genewiz, Inc.) and pcDNA3/EGFP/CCR5-3'UTR or mutant 3'UTR, whereas the control cells were transfected with pcDNA3/EGFP-CCR5-3'UTR or mutant 3'UTR only. The plasmid expressing red fluorescent protein (RFP) was transfected as the spike-in control. At $48 \mathrm{~h}$ after transfection, the cells were lysed using RIPA buffer, and the EGFP and RFP intensities were measured using an F-4500 fluorescence spectrophotometer (Hitachi, Ltd., Tokyo, Japan).

Statistical analysis. All data are presented as the mean \pm standard deviation, and the difference between groups was determined using the two-tailed Student's t-test. $\mathrm{P}<0.05$ was considered to indicate a statistically significant difference.

\section{Results}

CCR5 is upregulated in human cervical cancer tissues. In order to determined the role of CCR5 in cervical cancer, the mRNA expression levels of CCR5 were evaluated in 28 pairs of human cervical cancer samples and adjacent normal tissues using RT-qPCR. The results revealed that CCR5 mRNA expression was increased in the majority of cancer tissues compared with the matched normal control tissues (Fig. 1A). To further confirm the upregulation of CCR5, western blot analysis was performed to detect the CCR5 protein expression levels in samples 015 and 043 and confirmed that it was 
A

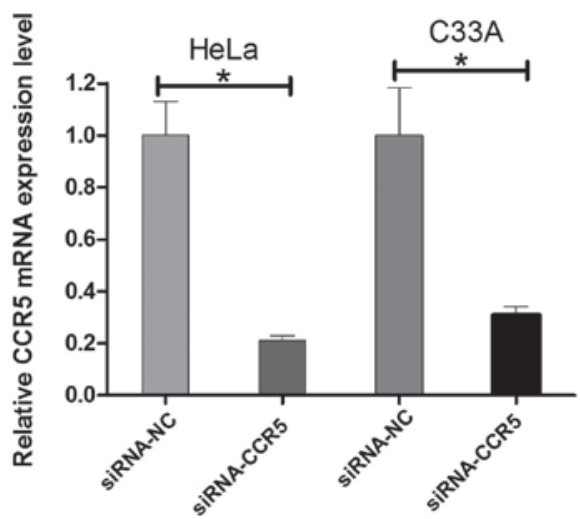

C

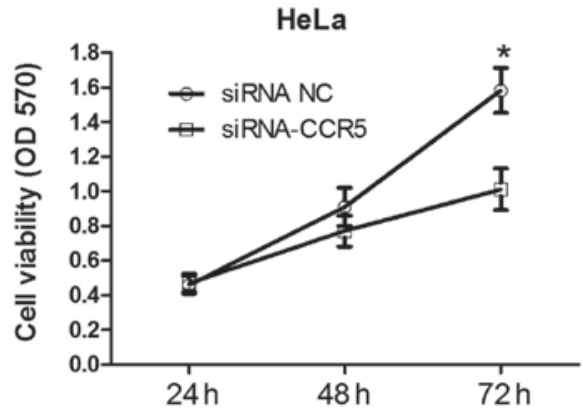

$\mathbf{E}$

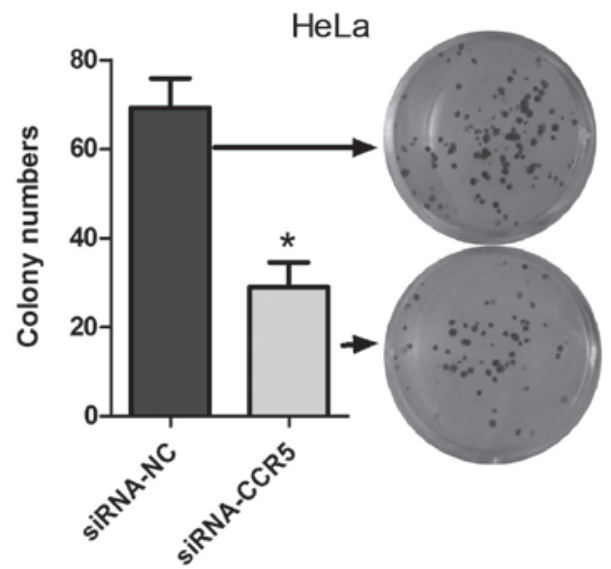

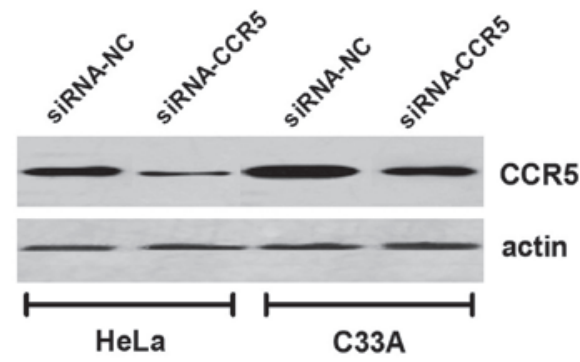

D

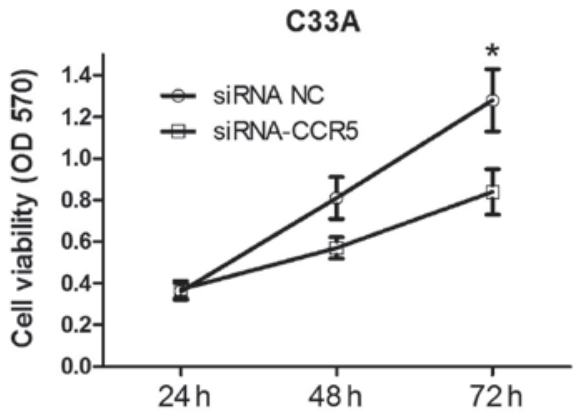

$\mathbf{F}$

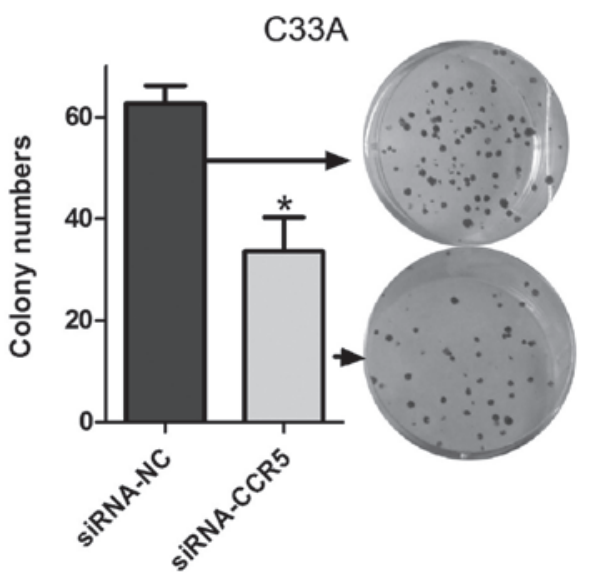

Figure 2. Downregulation of CCR5 levels inhibits HeLa and C33A cell growth. CCR5 expression level in HeLa and C33A cells was effectively reduced by siRNA-CCR5 transfection. (A) Reverse transcription-quantitative polymerase chain reaction and (B) western blot analysis were performed to detect CCR5 mRNA and protein expression, respectively. GAPDH was used for normalization. (C) HeLa and (D) C33A cells were transfected with the siRNA-CCR5 or control vector. MTT assays were used to determine the relative cellular proliferation at 48 and $72 \mathrm{~h}$. Cell absorption was measured at $570 \mathrm{~nm}$. The growth of the (E) HeLa and (F) C33A cells measured using colony formation assays. Data presented as the mean \pm standard deviation of three experiments. ${ }^{*} \mathrm{P}<0.05$ vs. siRNA-NC. CCR5, C-C chemokine receptor type 5; siRNA, small interfering RNA; NC, normal control; OD, optical density.

higher in the tumor tissue than in the adjacent normal tissue (Fig. 1B). These results indicate that CCR5 is upregulated in human cervical cancer, suggesting that CCR5 may exert an oncogenic effect in cervical cancer development.

Downregulation of CCR5 affects cervical cancer cell growth. On the basis of the results demonstrating that CCR5 is upregulated in cervical cancer cells, it was speculated that CCR5 may affect cervical cancer cell growth. Firstly, siRNA-CCR5 (for knockdown of CCR5) or an siRNA control vector were transfected into HeLa and C33A cells, and the mRNA and protein expression levels of CCR5 were evaluated using
RT-qPCR and western blot analysis. As shown in Fig. 2A and $\mathrm{B}, \mathrm{CCR} 5 \mathrm{mRNA}$ and protein expression levels in HeLa and $\mathrm{C} 33 \mathrm{~A}$ cells transfected with siRNA-CCR5 were evidently decreased compared with those in the cells transfected with the scrambled siRNA. Next, an MTT assay was performed to determine the effect of CCR5 on HeLa and C33A cell viability. The results indicate that cells transfected with siRNA-CCR5 had decreased cell viability at 48 and $72 \mathrm{~h}$ compared with the cells transfected with scramble siRNA (Fig. 2C and D). In addition, colony formation assays were performed to assess the effect of CCR5 on the long-term proliferative capacity of HeLa and C33A cells. As shown in Fig. 2E and F, the colony 
A

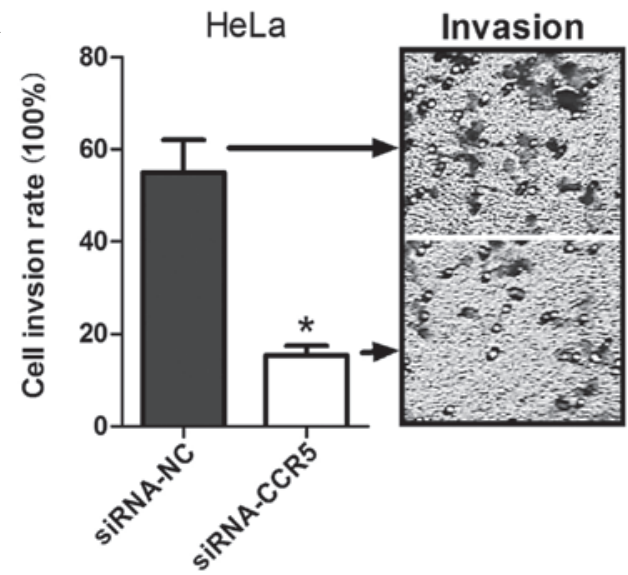

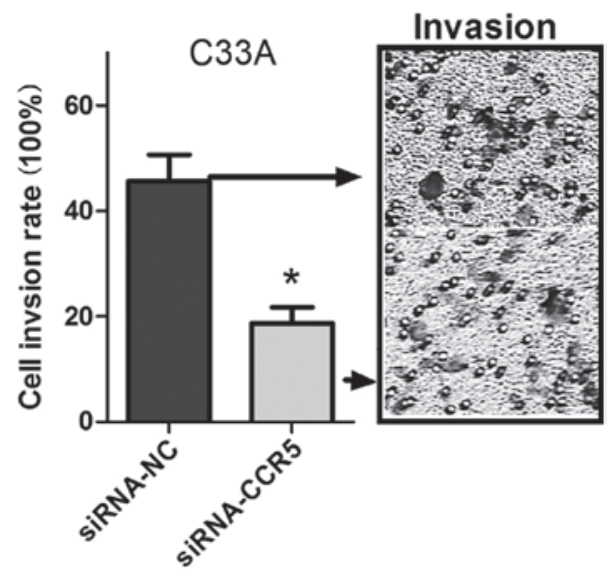

Figure 3. Downregulation of CCR5 inhibits the invasive ability of HeLa and C33A cells in vitro. Transwell invasion assays were performed using (A) HeLa and (B) C33A cells transfected with siRNA-CCR5 or control vector. The numbers of invasive cells were counted and representative images are shown. Data are presented as the mean \pm standard deviation of three experiments. ${ }^{*} \mathrm{P}<0.05$ vs. siRNA-NC. siRNA, small interfering RNA; CCR5, C-C chemokine receptor type 5; NC, normal control.

A

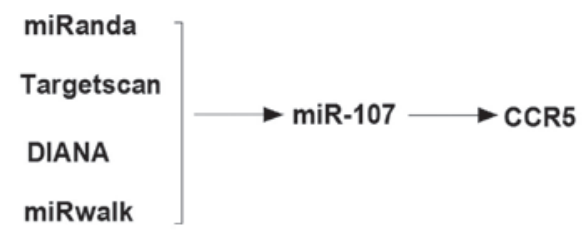

B
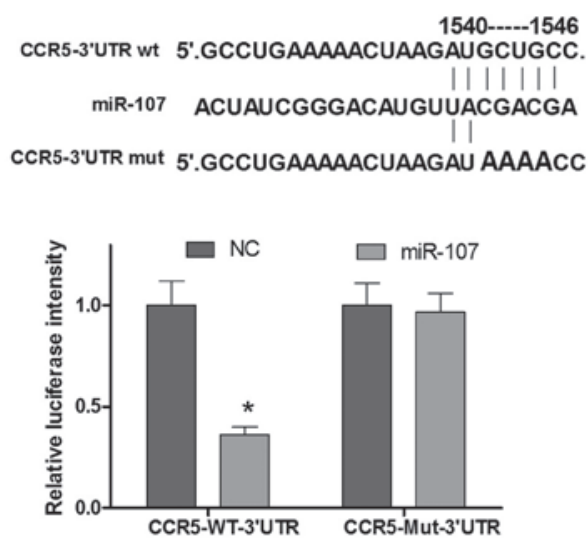

C

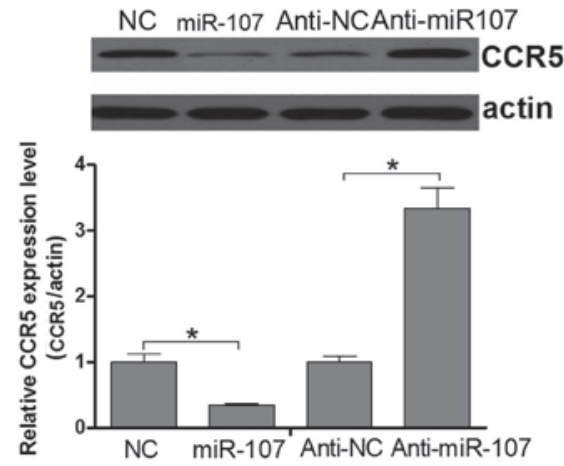

Figure 4. CCR5 is a direct target of miR-107. (A) CCR5 was identified as a potential target of miR-107 using bioinformatics. (B) Algorithms between miR-107 and the 3'UTR of CCR5, and the mutant CCR5 3'UTR, in which a number of nucleotides within binding sites were mutated. A luciferase reporter assay was performed to detect the effect of miR-107 on the luciferase intensity controlled by the 3'UTR of potential targets. (C) Western blot analysis was performed to detect the effect of miR-107 on the protein levels of CCR5 ( $\left.{ }^{*} \mathrm{P}<0.05\right)$. miR, microRNA; CCR5, C-C chemokine receptor type 5; UTR, untranslated region; wt, wild type; mut, mutant; NC, normal control; anti, antisense miR. number of HeLa and C33A cells treated with siRNA-CCR5 decreased by approximately half compared with the colony number in the control group. These results suggest that the downregulation of CCR5 is able to inhibit cervical cancer cell growth and proliferation.

Downregulation of CCR5 inhibits the invasion of cervical cancer cells. Previous studies have shown that CCR5 is associated with tumor cell invasiveness (15). Therefore, a cell invasion assay was performed to determine whether CCR5 was associated with cervical cancer cell invasion. Compared with the control group, the invasive cell number decreased $\sim 2$-fold in the HeLa and C33A cells transfected with siRNA-CCR5 (Fig. 3), which suggests that the downregulation of CCR5 is able to inhibit cervical carcinoma cell invasion.

miR-107 is a candidate regulator of CCR5 in cervical cancer cells. miRNAs function as tumor suppressors or oncogenes via the direct regulation of associated oncogenes or tumor suppressor genes. Tumor suppressor miRNAs are usually downregulated in tumors and may result in the reduced expression of tumor oncogenes and contribute to the tumorigenesis of cancers. The upregulation of CCR5 in cervical cancer tissues suggests that miRNAs may be crucially involved in the regulation of CCR5 in cervical cancer development. Improved specificity of miRNA prediction may be attained by the consensus of multiple algorithms . Therefore, four programs (Targetscan, miRanda, DIANA and miRwalk) were used in the present study to predict an miRNA sequence able to target the CCR5 3'UTR and regulate its expression. Finally, miR-107, the mRNA 3'UTR of which contains a putative CCR5 binding site (Fig. 4A), was identified as a candidate for directly targeting CCR5. This analysis is consistent with a model in which tumor suppressor miRNAs negatively regulate tumor oncogenes during tumor development. To confirm that CCR5 expression was directly regulated by miR-107, a luciferase reporter system was applied in HeLa cells. Fig. 4B shows that miR-107 was able to directly target the CCR5 mRNA 3'UTR. Next, whether the endogenous CCR5 was regulated by miR-107 in cervical 
cancer cells was investigated. The results of a western blot assay demonstrated that the CCR5 protein expression level was negatively regulated by miR-107 (Fig. 4C). Thus, it was concluded that CCR5 is negatively regulated by miR-107 in cervical cancer cells.

\section{Discussion}

CCR5 has been demonstrated to promote tumor growth in cancer cell in vitro and metastasis in a mouse model $(16,17)$. Furthermore, a prior study indicated that CCR5 heterozygous genotype $(+/ \Delta 32)$ may have a significant effect on the early stage of cervical cancer development (18). However, there is no direct evidence for the involvement of CCR5 in cervical cancer tumorigenesis. Therefore, the aim of the present study was to determine whether CCR5 participates in cervical cancer tumorigenesis. Firstly, CCR5 mRNA expression levels were evaluated using RT-qPCR and western blot analysis in cervical cancer tissues and matched adjacent normal control tissues. The results showed that the mRNA expression of CCR5 was upregulated in 21 cancer tissues compared with the matched normal tissues (28 pairs of specimens in total). Next, the effect of CCR5 on cervical cancer cell lines was investigated using an siRNA to knockdown CCR5. MTT and colony formation assays indicate that knockdown of CCR5 had an inhibitory effect on the growth of these cells. Thus, it may be speculated that CCR5 is able to promote cervical cancer proliferation. In addition, the results of cell invasion assays indicate that knockdown of CCR5 is able to inhibit $\mathrm{HeLa}$ and C33A cell invasion. Collectively, the present results suggest that CCR5 may function as an oncogene during cervical cancer development.

Accumulating evidence indicates that the downregulation of tumor suppressor miRNAs may be a common mechanism in the tumorigenesis of cervical cancer though target oncogenes. For example, miR-99a and $-99 \mathrm{~b}$ have been found to inhibit cervical cancer cell proliferation and invasion by targeting the mechanistic target of rapamycin signaling pathway (19). Another miRNA, miR-506 functions as a tumor suppressor by targeting the hedgehog signaling pathway transcription factor GLI3 in human cervical cancer cells (20). In the present study, CCR5 was upregulated in all of the cervical cancer tissues tested, indicating that it may serve an oncogenic function in tumorigenesis (Figs. 1-3). Therefore, in order to determine the association between the miRNA-mediated suppression of CCR5 and the expression of CCR5 in cervical cancer development, a bioinformatics approach was used in the present study to predict miRNAs that could bind to the CCR5-3'UTR Four independent miRNA target prediction algorithms indicated that miR-107 was a potential candidate. Furthermore, a luciferase reporter assay showed that miR-107 significantly decreased the luciferase activity of CCR5-3'UTR, while RT-qPCR and western blot analyses indicated that the miR-107 was able to directly repress endogenous CCR5 mRNA and protein expression. These results indicate that the upregulation of CCR5 may at least be partly attributed to downregulation of miR-107.

miR-107 has been a widely researched miRNA in the development of various types of cancer (21). To date, a number of studies have indicated the involvement of miR-107 in cell cycle arrest and growth suppression in lung and pancreatic cancer $(22,23)$. However, miR-107 has additionally been shown to promote invasiveness and metastatic dissemination in breast cancer cells (24). In addition, a previous study suggested that the upregulation of miRNA-107 exerts an inductive effect on the proliferation of human gastric cancer cells by targeting the transcription factor forkhead box protein $\mathrm{O} 1$ (25). Thus, it is apparent that miR-107 is able to function as a tumor suppressor or as an oncomiR, depending on the type of cell. The results of the present study indicate that miR-107 directly targets CCR5 and represses its expression in cervical cancer cells, which implies that the miR-107/CCR5 axis may contribute to the development of cervical cancer. However, the exact mechanism by which miR-107 affects the cervical cancer cell phenotype is unclear and requires further study.

In conclusion, CCR5 is overexpressed in cervical cancer cells and may function as a tumor oncogene. Furthermore, the knockdown of CCR5 was able to repress cervical cancer cell proliferation and invasion. In addition, it was identified and experimentally validated that miR-107 directly targets CCR5, potentially providing a molecular mechanism for the upregulation of CCR5 in cervical cancer. Therefore, miR-107 and CCR5 may be of use as novel therapeutic targets for the treatment of cervical cancer.

\section{Acknowledgements}

The authors of the present study would like to thank ZhiYou Biotechnology Co., Ltd. (Guangzhou, China) for their technical support.

\section{References}

1. Bedkowska GE, Ławicki S and Szmitkowski M: Molecular markers of carcinogenesis in the diagnostics of cervical cancer. Postepy Hig Med Dosw (Online) 63: 99-105, 2009 (In Polish).

2. Rajkumar T, Sabitha K, Vijayalakshmi N, Shirley S, Bose MV, Gopal $\mathrm{G}$ and Selvaluxmy G: Identification and validation of genes involved in cervical tumourigenesis. BMC Cancer 11: 80, 2011.

3. Hu X, Schwarz JK, Lewis JS Jr, Huettner PC, Rader JS, Deasy JO, Grigsby PW and Wang X: A microRNA expression signature for cervical cancer prognosis. Cancer Res 70: 1441-1448, 2010.

4. Ma YY, Wei SJ, Lin YC, Lung JC, Chang TC, Whang-Peng J, Liu JM, Yang DM, Yang WK and Shen CY: PIK3CA as an oncogene in cervical cancer. Oncogene 19: 2739-2744, 2000.

5. Su TH, Chang JG, Perng LI, Chang CP, Wei HJ, Wang NM and Tsai CH: Mutation analysis of the putative tumor suppressor gene PTEN/MMAC1 in cervical cancer. Gynecol Oncol 76: 193-199, 2000.

6. Ben-Baruch A, Michiel DF and Oppenheim JJ: Signals and receptors involved in recruitment of inflammatory cells. J Biol Chem 270: 11703-11706, 1995.

7. Cyster JG: Chemokines and the homing of dendritic cells to the T cell areas of lymphoid organs. J Exp Med 189: 447-450, 1999.

8. Cyster JG: Chemokines and cell migration in secondary lymphoid organs. Science 286: 2098-2102, 1999.

9. Luboshits G, Shina S, Kaplan O, Engelberg S, Nass D, Lifshitz-Mercer B, Chaitchik S, Keydar I and Ben-Baruch A: Elevated expression of the $\mathrm{CC}$ chemokine regulated on activation, normal $\mathrm{T}$ cell expressed and secreted (RANTES) in advanced breast carcinoma. Cancer Res 59: 4681-4687, 1999.

10. Mrowietz U, Schwenk U, Maune S, Bartels J, Küpper M, Fichtner I, Schröder JM and Schadendorf D: The chemokine RANTES is secreted by human melanoma cells and is associated with enhanced tumour formation in nude mice. $\mathrm{Br} \mathrm{J}$ Cancer 79 : 1025-1031, 1999. 
11. Robinson SC, Scott KA, Wilson JL, Thompson RG, Proudfoot AE and Balkwill FR: A chemokine receptor antagonist inhibits experimental breast tumor growth. Cancer Res 63: 8360-8365, 2003.

12. Ng-Cashin J, Kuhns JJ, Burkett SE, Powderly JD, Craven RR van Deventer HW, Kirby SL and Serody JS: Host absence of CCR5 potentiates dendritic cell vaccination. J Immunol 170: 4201-4208, 2003.

13. van Deventer HW, O'Connor W Jr, Brickey WJ, Aris RM, Ting JP and Serody JS: C-C chemokine receptor 5 on stromal cells promotes pulmonary metastasis. Cancer Res 65: 3374-3379, 2005

14. Son JH, Kong TW, Kim SH, Paek J, Chang SJ, Lee EJ and Ryu HS: Prediction of lymph node metastasis in patients with apparent early endometrial cancer. Obstet Gynecol Sci 58: 385-390, 2015

15. Wang J, He Q, Shao YG and Ji M: Chemokines fluctuate in the progression of primary breast cancer. Eur Rev Med Pharmacol Sci 17: 596-608, 2013.

16. Lin S, Wan S, Sun L, Hu J, Fang D, Zhao R, Yuan S and Zhang L: Chemokine $\mathrm{C}-\mathrm{C}$ motif receptor 5 and $\mathrm{C}-\mathrm{C}$ motif ligand 5 promote cancer cell migration under hypoxia. Cancer Sci 103: 904-912, 2012.

17. Mango RL, Wu QP, West M, McCook EC, Serody JS and van Deventer HW: C-C chemokine receptor 5 on pulmonary mesenchymal cells promotes experimental metastasis via the induction of erythroid differentiation regulator 1 . Mol Cancer Res 12: 274-282, 2014.

18. Singh H, Sachan R, Jain M and Mittal B: CCR5-Delta32 polymorphism and susceptibility to cervical cancer: Association with early stage of cervical cancer. Oncol Res 17: 87-91, 2008.
19. Wang L, Chang L, Li Z, Gao Q, Cai D, Tian Y, Zeng L and Li M: miR-99a and -99b inhibit cervical cancer cell proliferation and invasion by targeting mTOR signaling pathway. Med Oncol 31: 934, 2014.

20. Wen SY, Lin Y, Yu YQ, Cao SJ, Zhang R, Yang XM, Li J, Zhang YL, Wang YH, Ma MZ, et al: miR-506 acts as a tumor suppressor by directly targeting the hedgehog pathway transcription factor Gli3 in human cervical cancer. Oncogene 34: 717-725, 2015.

21. Zhou C, Li G, Zhou J, Han N, Liu Z and Yin J: miR-107 activates ATR/Chk1 pathway and suppress cervical cancer invasion by targeting MCL1. PLoS One 9: e111860, 2014.

22. Roldo C, Missiaglia E, Hagan JP, Falconi M, Capelli P, Bersani S, Calin GA, Volinia S, Liu CG, Scarpa A, et al: MicroRNA expression abnormalities in pancreatic endocrine and acinar tumors are associated with distinctive pathologic features and clinical behavior. J Clin Oncol 24: 4677-4684, 2006.

23. Takahashi Y, Forrest AR, Maeno E, Hashimoto T, Daub CO and Yasuda J: MiR-107 and MiR-185 can induce cell cycle arrest in human non small cell lung cancer cell lines. PLoS One 4: e6677, 2009.

24. Martello G, Rosato A, Ferrari F, Manfrin A, Cordenonsi M, Dupont S, Enzo E, Guzzardo V, Rondina M, Spruce T, et al: A microRNA targeting dicer for metastasis control. Cell 141: 1195-1207, 2010

25. Li F, Liu B, Gao Y, Liu Y, Xu Y, Tong W and Zhang A: Upregulation of microRNA-107 induces proliferation in human gastric cancer cells by targeting the transcription factor FOXO1. FEBS Lett 588: 538-544, 2014. 